\title{
EELS Evidence for Nascent Polymerization of Carbon and Silicon in Amorphization of SiC
}

\author{
Linn Hobbs ${ }^{1}$, Alexander Leide ${ }^{2}$ and $\mathrm{Ju} \mathrm{Li}^{3}$ \\ ${ }^{1}$ Massachusetts Institute of Technology, Belmont, Massachusetts, United States, ${ }^{2}$ Universisty of Oxford, \\ Oxford, England, United Kingdom, ${ }^{3}$ Massachusetts Institute of Technology, Cambridge, Massachusetts, \\ United States
}

Silicon carbide $(\mathrm{SiC})$ is an important refractory ceramic material, whose excellent high-temperature performance, high thermal conductivity, low thermal expansion, wide-band semiconduction, and relative chemical inertness render it useful for a variety of high-temperature structural applications, hightemperature semiconductor device realizations, high-strength fiber composites, and nuclear materials needs [1]. In nuclear application, its low-neutron absorption cross-section and inherently low level of longlived radioisotopes produced through nuclear transmutations present particular advantages for structural and cladding components of fission reactors, blanket components for fusion reactors, encapsulation media for storing nuclear waste, and advanced fuel-pellet designs. SiC crystallizes in a cubic polymorph with zincblende structure ( $\beta$-SiC, or " $3 \mathrm{C}$ " in the Ramsdell notation), a hexagonal polymorph ( $\alpha-\mathrm{SiC}$, or " $6 \mathrm{H}$ ") with wurtzite structure, and a large family of longer-period polytypes. Its crystalline states are, however, susceptible to "amorphization" (loss of medium-range order consistent with periodic translation) by atomic displacements [2] induced by fast neutrons and atom recoils, energetic ions (including those used in ion implantation in semiconductor applications), and fast electrons (even the $100 \mathrm{keV}$ electrons in an electron microscope [3]) below critical radiation temperatures $\left(\mathrm{T}_{\mathrm{c}} \sim 500-700 \mathrm{~K}\right)$ that are not far above room temperature. The amorphization results in macroscopic swelling, significant changes in mechanical properties, and alteration of electronic states [3].

A fundamental topological quandary is that $\mathrm{SiC}$ cannot exist in an aperiodic state if the Si-C connectivity and stoichiometry are maintained [4,5] (i.e. assemblies of corner-sharing [ $\left.\mathrm{SiC}_{4}\right]$ or [CSi4] tetraheda), yet its experimental susceptibility to displacement-induced amorphization by radiation is anomalously close to that for $\mathrm{Si}$ and not far from that for silicas [6]. This fact has led one of the present authors to propose that a necessary condition for amorphization of $\mathrm{SiC}$ is a critical level of induced chemical disorder [7]that is, the accumulation of a critical density of $\mathrm{C}-\mathrm{C}$ (or $\mathrm{Si}-\mathrm{Si}$ ) homonuclear bonds with a chemicaldisorder parameter defined as $\chi=\mathrm{n}(\mathrm{C}-\mathrm{C}) / \mathrm{n}(\mathrm{Si}-\mathrm{C})>0.3$.

The present contribution explores the use of ab initio molecular dynamics (MD) computational modeling (VASP, 216 atoms with periodic boundary conditions, thermostatted in the canonical assembly, with a 1 fs timestep [8]. Random atom swaps were made in an initial 3C $\beta$-SiC crystalline assembly to induce initial disorder parameters from $\chi_{0}=0.09$ to $\chi_{0}=0.58$, which after relaxation resulted in disorder parameters from $\chi=0.09$ to $\chi=0.43$. A crystalline assembly was also quenched from a virtual melt state at $6000 \mathrm{~K}$ (virtual, because real $\mathrm{SiC}$ sublimes rather than melts) and relaxed, resulting in a relaxed disorder parameter of $\chi=0.24$. The connectivities of all resulting assemblies (including the initial crystalline assembly) were evaluated using connectivity topology approaches (see [5]) involving local cluster analysis, and local cluster primitive-ring size distributions. Atom-swap structures with relaxed $\chi>0.30$ were all assessed to be amorphous, as was the quenched structure with $\chi=0.24$. 
An experimental wafer sample of single-crystal $6 \mathrm{H} \alpha$-SiC was amorphized by ion-irradiating with 1.35 $\mathrm{MeV} \mathrm{Fe}{ }^{+}$ions at a flux of $6.8 \times 10^{15}$ ions $/ \mathrm{m}^{2}$ s to a total fluence of $1 \times 10^{20}$ ions $/ \mathrm{m}^{2}$, with the ion beam oriented $5-8^{\circ}$ from the [0001] wafer normal. A cross-section TEM specimen was milled out using a focused ion beam and lift-out technique. Electron energy-loss spectra were obtained using a Gatan GIF 2001 energy filter interfaced with a JEOL JEM-2010F field-emission analytical TEM operating at 200 $\mathrm{kV}$. Analysis focused on the C K-edge near-edge energy-loss structure (Fig. 1) from unirradiated, unamorphized, and amorphized regions. Comparison spectra were simulated using the FEFF9 code from the initial 3C-SiC ab initio crystalline structure, from each of the $a b$ initio modeled assemblies with increasing chemical disorder, and from the quenched ab initio modeled assembly (also Fig. 1). The feature of interest was the peak at $\sim 284 \mathrm{eV}$ loss, attributable to the $\pi^{*}$ transition expected from $\mathrm{sp}^{2}$ bond hybridization found in 3-coordinated $\mathrm{C}$ atoms with co-planar bond angles of $\sim 120^{\circ}$, as found for example in graphite [8]. This spectral feature was found in the ion-amorphized regions of the experimental sample, in the melt-quenched and relaxed $a b$ initio modeled assembly, and in the atom-swap ab initio modeled and relaxed assemblies with $\chi>0.30$ (the $\chi=0.54$ atom-swap assembly spectrum is illustrated in Fig. 1), but not in the unirradiated experimental crystal or in the ab initio modeled initial perfect crystal. The implication is that this spectral feature is an indicator for amorphous $\mathrm{SiC}$.

Some atomistic construct beyond (easily recoverable) short-range chemical disorder must be preventing disordered $\mathrm{SiC}$ from relaxing back into an ordered crystalline state (or even to a quasi-crystalline chemically disordered state with random site occupancies (by an "average" atom). The ab initio amorphous models, probed for such constructs, reveal (Fig. 2) presence of short carbon atom chains (i.e. $\mathrm{C}-\mathrm{C}-\mathrm{C}$ homonuclear arrays), in some cases co-planar with $120^{\circ}$ bond angles (i.e. proto-graphitic). Localized graphitic networks were observed in the melt-quench models and also limited [Si4] tetrahedral silicon networks. These features constitute local two-phase decomposition, clearly providing strongly stochastic impediments to recrystallization of initially disordered assemblies. The conclusion, confirmed by combining ab initio modeling with experimental and simulated ELNES, is that a threshold chemical disorder leads to limited formation of more extended homonuclear networks that can maintain disordered $\mathrm{SiC}$ in an aperiodic state. 


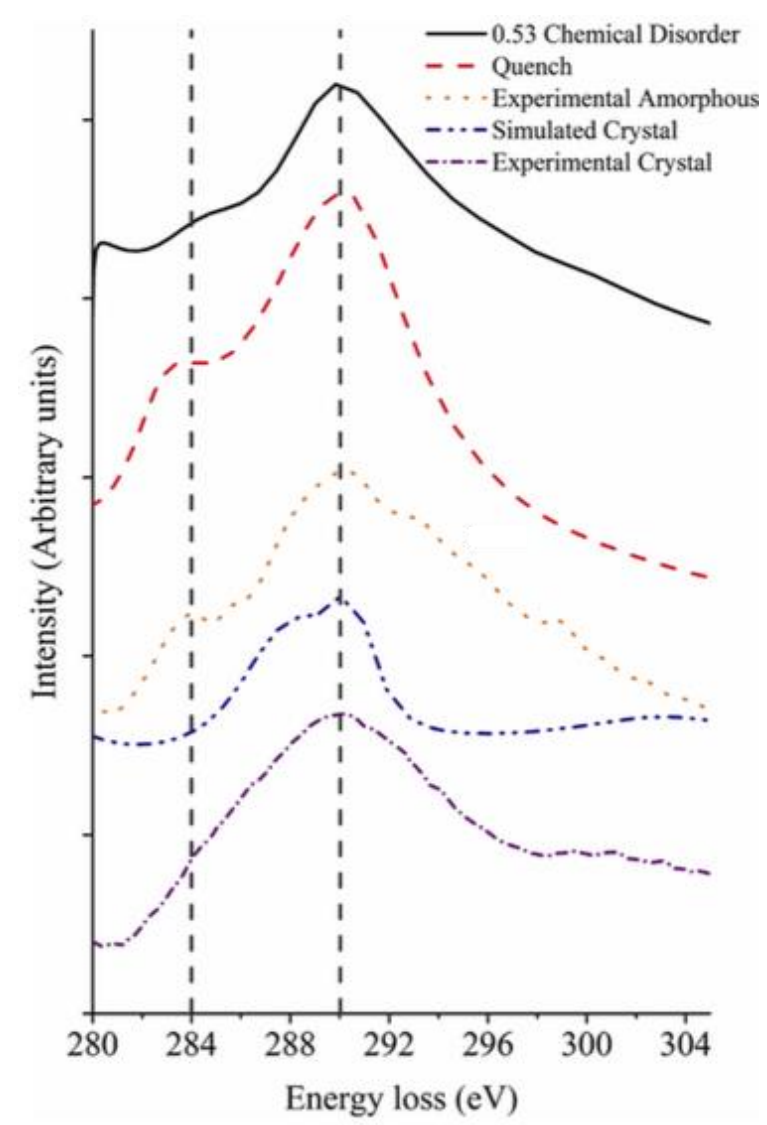

Figure 1. C K-edge ELNES spectra from modeled and experimental SiC, and from amorphized $\mathrm{SiC}$ derived from atom-swapping or melt-quenching ab initio models or from ion irradiation. The peak at 284 $\mathrm{eV}$ corresponds to the $\pi^{*}$ transition in $\mathrm{sp}^{2}$-bonded $\mathrm{C}$.

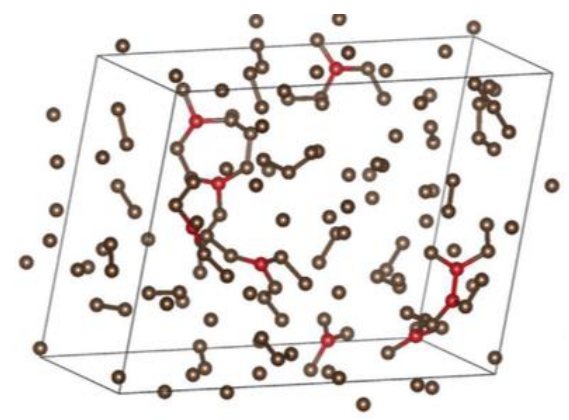

(a)

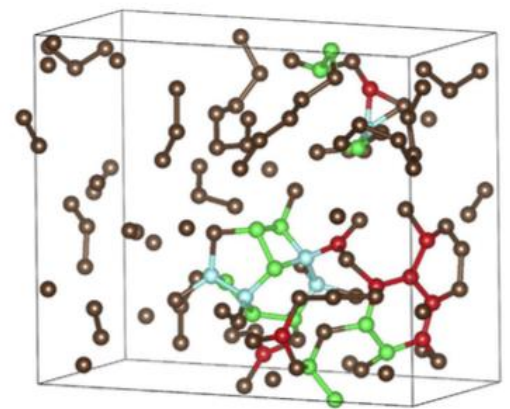

(b) 
Figure 2. Homonuclear C-C-C chains and incipient planar graphitic networks developed in a) $\chi=0.54$ atom-swapped $a b$ initio $\mathrm{SiC}$ model; b) melt quenched $a b$ initio $\mathrm{SiC}$ model.

\section{References}

1. C. F. Zhe, Silicon Carbide Materials, Processing and Devices (CRC Press, Boca Raton, FL, 2003).

2. S. Muto, T. Tanabe, Local structures and damage processes of electron-irradiated SiC studied with transmission electron microscopy and electron energy-loss spectroscopy, J. Appl. Phys. 93 (2003) 37653775 .

3. Y. Katoh, L. L. Snead, I. Szlufarska, W. J. Weber, Radiation effects in SiC for nuclear structural applications, Current Opin. Solid State Mater. Sci. 16 (2012) 143-152.

4. L. W. Hobbs, The role of topology and geometry in the radiation-induced amorphization of network structures, J. Non-Cryst. Solids 182 (1995) 27-39.

5. L. W. Hobbs, Topological approaches to the structure of crystalline and amorphous atom assemblies, in Engineering of Crystalline Materials Properties, ed. J. J. Novoa, D. Braga, L. Addadi (Springer, 2008) pp. 193-230.

6. L. W. Hobbs, C. E. Jesurum, B. Berger, Rigidity constraints in the amorphization of single- and multiply-polytopic structures, in Rigidity Theory and Applications, ed. P. M. Duxbury, M. F. Thorpe (Plenum, New York, 1999) pp. 191-216.

7. X. Yuan, L. W. Hobbs, Modeling chemical and topological disorder in irradiation-amorphized silicon carbide, Nucl. Instrum. Methods Phys. Res. B 192 (2002) 74-82.

8. A. J. Leide, L. W. Hobbs, Z. Wang, D. Chen, L. Shao, J. Li, The role of chemical disorder and structural freedom in radiation-induced amorphization of silicon carbide. deduced from electron spectroscopy and ab intio simulations, J. Nucl. Mater. 514 (2019) 299-310.

9. F. Finocchi, G. Galli, M. Parrinello, C. M. Bertoni, Structural properties of amorphized SiC via ab initio molecular dynamics, J. Non-Cryst. Solids 137-138 Part I (1999) 153-156; also, Phys. Rev. Lett. 68 (1992) 3044-3047. 